\title{
PCADAPT : AN R PACKAGE TO PERFORM GENOME SCANS FOR SELECTION BASED ON PRINCIPAL COMPONENT ANALYSIS.
}

\author{
Keurcien Luu ${ }^{1} \&$ Eric Bazin ${ }^{2} \&$ Michael G. B. Blum ${ }^{1}$ \\ 1 Université Grenoble Alpes, CNRS, Laboratoire TIMC-IMAG, UMR 5525, France. \\ ${ }^{2}$ Université Grenoble Alpes, CNRS, Laboratoire d'Ecologie Alpine UMR 5553, France
}

\section{Abstract}

(1) on population genomic data. It assumes that candidate markers are outliers with respect to how they are related to population structure. Because population structure is ascertained with principal component analysis, the package is fast and works with large-scale data. It can handle missing data and pooled sequencing data. By contrast to populationbased approaches, the package handle admixed individuals and does not require grouping individuals into populations. Since its first release, pcadapt has evolved both in terms of statistical approach and software implementation. We present results obtained with robust Mahalanobis distance, which is a new statistic for genome scans available in the 2.0 and later versions of the package. When hierarchical population structure occurs, Mahalanobis distance is more powerful than the communality statistic that was implemented in the first version of the package. Using simulated data, we compare pcadapt to other software for genome scans (BayeScan, hapflk, OutFLANK, sNMF). We find that the proportion of false discoveries is around a nominal false discovery rate set at $10 \%$ with the exception of BayeScan that generates $40 \%$ of false discoveries. We also find that the power of BayeScan is severely impacted by the presence of admixed individuals whereas pcadapt is not impacted. Last, we find that pcadapt and hapflk are the most powerful software in scenarios of population divergence and range expansion. Because pcadapt handles next-generation sequencing data, it is a valuable tool for data analysis in molecular ecology.

Keywords. population genetics, R package, outlier detection, Mahalanobis distance, principal component analysis. 


\section{Introduction}

Looking for variants with unexpectedly large differences of allele frequencies between populations is a common approach to detect signals of natural selection (Lewontin and Krakauer, 1973). When variants confer a selective advantage in the local environment, allele frequency changes are triggered by natural selection leading to unexpectedly large differences of allele frequencies between populations. To detect variants with large differences of allele frequencies, numerous test statistics have been proposed, which are usually based on chi-square approximations of $F_{S T}$-related test statistics (François et al., 2016).

Statistical approaches for detecting selection should address several challenges. The first challenge is to account for hierarchical population structure that arises when genetic differentiation between populations is not identical between all pairs of populations. Statistical tests based on $F_{S T}$ that do not account for hierarchical structure, when it occurs, generate a large excess of false positive loci (Bierne et al., 2013; Excoffier et al., 2009).

A second challenge arises because approaches based on $F_{S T}$-related measures require to group individuals into populations, although defining populations is a difficult task (Waples and Gaggiotti, 2006). Individual sampling may not be population-based but based on more continuous sampling schemes (Lotterhos and Whitlock, 2015). Additionally assigning an admixed individual to a single population involves some arbitrariness because different regions of its genome might come from different populations (Pritchard et al., 2000). Several individual-based methods of genome scans have already been proposed to address this challenge and they are based on related techniques of multivariate analysis including principal component analysis (PCA), factor models, and non-negative matrix factorization (Duforet-Frebourg et al., 2014; Hao et al., 2016; Galinsky et al., 2016; Chen et al., 2016; Duforet-Frebourg et al., 2016; Martins et al., 2016).

The last challenge arises from the nature of multilocus datasets generated from next generation sequencing platforms. Because datasets are massive with a large number of molecular markers, Monte Carlo methods usually implemented in Bayesian statistics may be prohibitively slow (Lange et al., 2014). Additionally, next generation sequencing data may contain a substantial proportion of missing data that should be accounted for (Arnold et al., 2013; Gautier et al., 2013).

To address the aforementioned challenges, we have developed the software pcadapt and the $\mathrm{R}$ package pcadapt. The software pcadapt is now deprecated and the $\mathrm{R}$ package only is 
maintained. pcadapt assumes that markers excessively related with population structure are candidates for local adaptation. Since its first release, pcadapt has substantially evolved both in terms of statistical approach and software implementation (Table 1).

The first release of pcadapt was a command line $\mathrm{C}$ software. It implemented a Monte Carlo approach based on a Bayesian factor model (Duforet-Frebourg et al., 2014). The test statistic for outlier detection was a Bayes factor. Because Monte Carlo methods can be computationally prohibitive with massive NGS data, we then developed an alternative approach based on PCA. The first statistic based on PCA was the communality statistic, which measures the percentage of variation of a SNP explained by the first $K$ principal components (Duforet-Frebourg et al., 2016). It was initially implemented with a commandline $\mathrm{C}$ software (the pcadapt fast command) before being implemented in the pcadapt $\mathrm{R}$ package. We do not maintain $\mathrm{C}$ versions of pcadapt anymore. The whole analysis that goes from reading genotype files to detecting outlier SNPs can now be performed in $\mathrm{R}$ ( $\mathrm{R}$ Core Team, 2015).

The 2.0 and following versions of the $\mathrm{R}$ package implement a more powerful statistic for genome scans. The test statistic is a robust Mahalanobis distance. A vector containing $K z$-scores measures to what extent a SNP is related to the first $K$ principal components. The Mahalanobis distance is then computed for each SNP to detect outliers for which the vector of $z$-scores do not follow the distribution of the main bulk of points. The term robust refers to the fact that the estimators of the mean and of the covariance matrix of $z$, which are required to compute Mahalanobis distances, are not sensitive to the presence of outliers in the dataset (Maronna and Zamar, 2012). In the following, we provide a comparison of statistical power that shows that Mahalanobis distance provides more powerful genome scans compared to the communality statistic and to the Bayes factor that were implemented in previous versions of pcadapt.

In addition to comparing the different test statistics that were implemented in pcadapt, we compare statistic performance obtained with the 3.0 version of pcadapt and with other software of genome scans. We use simulated data to compare software in terms of false discovery rate (FDR) and statistical power. We consider data simulated under different demographic models including island model, divergence model and range expansion. To perform comparisons, we include software that require to group individuals into populations : BayeScan (Foll and Gaggiotti, 2008), the $F_{L K}$ statistic as implemented in the hapflk software (Bonhomme et al., 2010), and OutFLANK that provides a robust estima- 
tion of the null distribution of a $F_{S T}$ test statistic (Whitlock and Lotterhos, 2015). We additionally consider the $s N M F$ software that implements another individual-based test statistic for genome scans (Frichot et al., 2014; Martins et al., 2016).

\section{Statistical and Computational approach}

\section{Input data}

The $\mathrm{R}$ package can handle different data formats for the genotype data matrix. In the version 3.0 that is currently available on CRAN, the package can handle genotype data files in the $v c f$, ped and lfmm formats. In addition, the package can also handle a pcadapt format, which is a text file where each line contains the allele counts of all individuals at a given locus. When reading a genotype data matrix with the read.pcadapt function, a .pcadapt file is generated, which contains the genotype data in the pcadapt format.

\section{Choosing the number of principal components}

In the following, we denote by $n$ the number of individuals, by $p$ the number of genetic markers, and by $G$ the genotype matrix that is composed of $n$ lines and $p$ columns. The genotypic information at locus $j$ for individual $i$ is encoded by the allele count $G_{i j}$, $1 \leq i \leq n$ and $1 \leq j \leq p$, which is a value in 0,1 for haploid species and in $0,1,2$ for diploid species.

First, we normalize the genotype matrix columnwise. For diploid data, we consider the usual normalization in population genomics where $\tilde{G}_{i j}=\left(G_{i j}-p_{j}\right) /\left(2 \times p_{j}\left(1-p_{j}\right)\right)^{1 / 2}$, and $p_{j}$ denotes the minor allele frequency for locus $j$ (Patterson et al., 2006). The normalization for haploid data is similar except that the denominator is given by $\left(p_{j}\left(1-p_{j}\right)\right)^{1 / 2}$.

Then, we use the normalized genotype matrix $\tilde{G}$ to ascertain population structure with PCA (Patterson et al., 2006). The number of principal components to consider is denoted $K$ and is a parameter that should be chosen by the user. In order to choose $K$, we recommend to consider the graphical approach based on the scree plot (Jackson, 1993). The scree plot displays the eigenvalues of the covariance matrix $\Omega$ in descending order. Up to a constant, eigenvalues are proportional to the proportion of variance explained by each principal component. The eigenvalues that correspond to random variation lie on a straight line whereas the ones corresponding to population structure depart from the 
line. We recommend to use Cattell's rule that states that components corresponding to eigenvalues to the left of the straight line should be kept (Cattell, 1966).

\section{Test statistic}

We now detail how the package computes the test statistic. We consider multiple linear regressions by regressing each of the $p$ SNPs by the $K$ principal components $X_{1}, \ldots, X_{K}$

$$
G_{j}=\sum_{k=1}^{K} \beta_{j k} X_{k}+\epsilon_{j}, j=1, \ldots, p
$$

where $\beta_{j k}$ is the regression coefficient corresponding to the $j$-th SNP regressed by the $k$-th principal component, and $\epsilon_{j}$ is the residuals vector. To summarize the result of the regression analysis for the $j$-th SNP, we return a vector of $z$-scores $z_{j}=\left(z_{j 1}, \ldots, z_{j K}\right)$ where $z_{j k}$ corresponds to the $z$-score obtained when regressing the $j$-th SNP by the $k$-th principal component.

The next step is to look for outliers based on the vector of $z$-scores. We consider a classical approach in multivariate analysis for outlier detection. The test statistic is a robust Mahalanobis distance $D$ defined as

$$
D_{j}^{2}=\left(z_{j}-\bar{z}\right)^{T} \Sigma^{-1}\left(z_{j}-\bar{z}\right)
$$

where $\Sigma$ is the $(K \times K)$ covariance matrix of the $z$-scores and $\bar{z}$ is the vector of the $K z$-score means (Maronna and Zamar, 2012). When $K>1$, the covariance matrix $\Sigma$ is estimated with the Orthogonalized Gnanadesikan-Kettenring method that is a robust estimate of the covariance able to handle large-scale data (Maronna and Zamar, 2012) (covRob function of the robust $\mathrm{R}$ package). When $K=1$, the variance is estimated with another robust estimate (cov.rob function of the MASS R package).

\section{Genomic Inflation Factor}

To perform multiple hypothesis testing, Mahalanobis distances should be transformed into $p$-values. If the $z$-scores were truly multivariate Gaussian, the Mahalanobis distances $D$ should be chi-square distributed with $K$ degrees of freedom. However, as usual for genome scans, there are confounding factors that inflate values of the test statistic and 
that would lead to an excess of false positives (François et al., 2016). To account for the inflation of test statistics, we divide Mahalanobis distances by a constant $\lambda$ to obtain a statistic that can be approximated by a chi-square distribution with $K$ degrees of freedom. This constant is estimated by the genomic inflation factor defined here as the median of the Mahalanobis distances divided by the median of the chi-square distribution with $K$ degrees of freedom (Devlin and Roeder, 1999).

\section{Control of the false discovery rate (FDR)}

Once $p$-values are computed, there is a problem of decision-making related to the choice of a threshold for $p$-values. We recommend to use the FDR approach where the objective is to provide a list of candidate genes with an expected proportion of false discoveries smaller than a specified value. For controlling the FDR, we consider the $q$ value procedure as implemented in the qvalue $\mathrm{R}$ package that is less conservative than Bonferroni or Benjamini-Hochberg correction (Storey and Tibshirani, 2003). The qvalue R package transforms the $p$-values into $q$-values and the user can control a specified value $\alpha$ of FDR by considering as candidates the SNPs with $q$-values smaller than $\alpha$.

\section{Numerical computations}

PCA is performed using a $\mathrm{C}$ routine that allows to compute scores and eigenvalues efficiently with minimum RAM access (Duforet-Frebourg et al., 2016). Computing the covariance matrix $\Omega$ is the most computationally demanding part. To provide a fast routine, we compute the $n \times n$ covariance matrix $\Omega$ instead of the much larger $p \times p$ covariance matrix. We compute the covariance $\Omega$ incrementally by adding small storable covariance blocks successively. Multiple linear regression is then solved directly by computing an explicit solution, written as a matrix product. Using the fact that the $(n, K)$ score matrix $X$ is orthogonal, the $(p, K)$ matrix $\hat{\beta}$ of regression coefficients is given by $G^{T} X$ and the $(n, p)$ matrix of residuals is given by $G-X X^{T} G$. The $z$-scores are then computed using the standard formula for multiple regression

$$
z_{j k}=\hat{\beta}_{j k} \sqrt{\frac{\sum_{i=1}^{n} x_{i k}^{2}}{\sigma_{j}^{2}}},
$$


where $\sigma_{j}^{2}$ is an estimate of the residual variance for the $j^{t h} \mathrm{SNP}$, and $x_{i k}$ is the score of $k^{\text {th }}$ principal component for the $i^{\text {th }}$ individual.

\section{Missing data}

Missing data should be accounted for when computing principal components and when computing the matrix of $z$-scores. There are many methods to account for missing data in PCA and we consider the pairwise covariance approach (Dray and Josse, 2015). It consists in estimating the covariance between each pair of individuals using only the markers that are available for both individuals. To compute $z$-scores, we account for missing data in formula (3). The term in the numerator $\sum_{i=1}^{n} x_{i k}^{2}$ depends on the quantity of missing data. If there are no missing data, it is equal to 1 by definition of the scores obtained with PCA. As the quantity of missing data grows, this term and the $z$-score decrease such that it becomes more difficult to detect outlier markers.

\section{Pooled sequencing}

When data are sequenced in pool, the Mahalanobis distance is based on the matrix of allele frequency computed in each pool instead of the matrix of $z$-scores.

\section{Materials and Methods}

\section{Simulated data}

We simulated SNPs under an island model, under a divergence model and we downloaded simulations of range expansion (Lotterhos and Whitlock, 2015). All data we simulated were composed of 3 populations, each of them containing 50 sampled diploid individuals (Table 2). SNPs were simulated assuming no linkage disequilibrium. SNPs with minor allele frequencies lower than $5 \%$ were discarded from the datasets. The mean $F_{S T}$ for each simulation was comprised between $5 \%$ and $10 \%$. Using the simulations based on a island and a divergence model, we also created datasets composed of admixed individuals. We assumed that an instantaneous admixture event occurs at the present time so that all sampled individuals are the results of this admixture event. Admixed individuals were 
generated by drawing randomly admixture proportions using a Dirichlet distribution of parameter $(\alpha, \alpha, \alpha)$ ( $\alpha$ ranging from 0.005 to 1 depending on the simulation).

\section{Island model}

We used $m s$ to create simulations under an island model (Fig SI1). We set a lower migration rate for the 50 adaptive SNPs compared to the 950 neutral ones to mimick diversifying selection (Bazin et al., 2010). For a given locus, migration from population $i$ to $j$ was specified by choosing a value of the effective migration rate that is set to $M_{\text {neutral }}=10$ for neutral SNPs and to $M_{\text {adaptive }}$ for adaptive ones. We simulated 35 datasets in the island model with different strengths of selection, where the strength of selection corresponds to the ratio $M_{\text {neutral }} / M_{\text {adaptive }}$ that varies from 10 to 1,000. The $m s$ command lines for neutral and adaptive SNPs are given by $\left(M_{\text {adaptive }}=0.01\right.$ and $\left.M_{\text {neutral }}=10\right)$

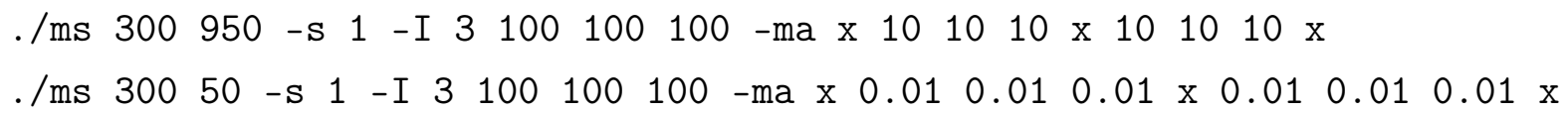

\section{Divergence model}

To perform simulations under a divergence model, we used the package simuPOP, which is an individual-based population genetic simulation environment (Peng and Kimmel, 2005). We assumed that an ancestral panmictic population evolved during 20 generations before splitting into two subpopulations. The second subpopulation then split into subpopulations 2 and 3 at time $T>20$. All 3 subpopulations continued to evolve until 200 generations have been reached, without migration between them (Figure SI1). A total of 50 diploid individuals were sampled in each population. Selection only occured in the branch associated with population 2 and selection was simulated by assuming an additive model (fitness is equal to $1-2 s, 1-s, 1$ depending on the genotypes). We simulated a total of 3,000 SNPs comprising of 100 adaptive ones for which the selection coefficient is of $s=0.1$.

\section{Range expansion}

We downloaded in the Dryad Digital Repository six simulations of range expansion with two glacial refugia (Lotterhos and Whitlock, 2015). Adaptation occurred during the recolonization phase of the species range from the two refugia. We considered six different 
simulated data with 30 populations and a number of sampled individual per location that varies from 20 to 60 .

\section{Parameter settings for the different software}

When using hapflk, we set $K=1$ that corresponds to the computation of the $F L K$ statistic. When using BayeScan and OutFLANK, we used the default parameter values. For $s N M F$, we used $K=3$ for the island and divergence model and $K=5$ for range expansion as indicated by the cross-entropy criterion. The regularization parameter of $s N M F$ was set to $\alpha=1000$. For $s N M F$ and hapflk, we used the genomic inflation factor to recalibrate $p$-values. When using population-based methods with admixed individuals, we assigned each individual to the population with maximum amount of ancestry.

\section{Results}

\section{Choosing the number of principal components}

We evaluate Cattell's graphical rule to choose the number of principal components. For the island and divergence model, the choice of $\mathrm{K}$ is evident (Figure 1 ). For $K \geq 3$, the eigenvalues follow a straight line. As a consequence, Cattell's rule indicates $K=2$, which is expected because there are 3 populations (Patterson et al., 2006). For the model of range expansion, applying Cattell's rule to choose $K$ is more difficult (Figure 1). Ideally, the eigenvalues that correspond to random variation lie on a straight line whereas the ones corresponding to population structure depart from the line. However, there is no obvious point at which eigenvalues depart from the straight line. Choosing a value of $K$ between 5 and 8 is compatible with Cattell's rule. Using the package qvalue to control $10 \%$ of FDR, we find that the actual proportion of false discoveries as well as statistical power is weakly impacted when varying the number of principal components from $K=5$ to $K=8$ (Figure SI2).

\section{An example of genome scans performed with pcadapt}

To provide an example of results, we apply pcadapt with $K=6$ in the model of range expansion. Population structure captured by the first 2 principal components is displayed 
in Figure 2. $P$-values are well calibrated because they are distributed as a mixture of a uniform distribution and of a peaky distribution around 0 , which corresponds to outlier loci (Figure 2). Using a FDR threshold of $10 \%$ with the qvalue package, we find 122 outliers among 10,000 SNPs, resulting in $23 \%$ actual false discoveries and a power of $95 \%$.

\section{Control of the false discovery rate}

We evaluate to what extent using the packages pcadapt and qvalue control a FDR set at $10 \%$ (Figure 3). All SNPs with a $q$-value smaller than $10 \%$ were considered as candidate SNPs. For the island model, we find that the proportion of false discoveries is $8 \%$ and it increases to $10 \%$ when including admixture. For the divergence model, the proportion of false discoveries is $11 \%$ and it increases to $22 \%$ when including admixture. The largest proportion of false discoveries is obtained under range expansion and is equal to $25 \%$.

We then evaluate the proportion of false discoveries obtained with BayeScan, hapflk, OutFLANK, and sNMF (Figure 3). We find that hapflk is the most conservative approach $(\mathrm{FDR}=6 \%)$ followed by OutFLANK and pcadapt $(\mathrm{FDR}=11 \%)$. The software $s N M F$ is more liberal (FDR $=19 \%$ ) and BayeScan generates the largest proportion of false discoveries $(\mathrm{FDR}=41 \%)$. When not recalibrating the $p$-values of hapflk, we find that the test is even more conservative (results not shown). For all software, the range expansion scenario is the one that generates the largest proportion of false discoveries. Proportion of false discoveries under range expansion ranges from $22 \%$ (OutFLANK) to $93 \%$ (BayeScan).

\section{Statistical power}

To provide a fair comparison between methods and software, we compare statistical power for equal values of the observed proportion of false discoveries. Then we compute statistical power averaged over observed proportion of false discoveries ranging from $0 \%$ to $50 \%$.

We first compare statistical power obtained with the different statistical methods that have been implemented in pcadapt (Table 1). For the island model, Bayes factor, communality statistic and Mahalanobis distance have similar power (Figure 4). For the divergence model, the power obtained with Mahalanobis distance is $20 \%$ whereas the power obtained with the communality statistic and with the Bayes factor is respectively $4 \%$ and $2 \%$ (Figure 4). Similarly, for range expansion, the power obtained with Mahalanobis distance 
is $46 \%$ whereas the power obtained with the communality statistic and with the Bayes factor is $34 \%$ and $13 \%$. We additionally investigate to what extent increasing sample size in each population from 20 to 60 individuals affects power. For range expansion, the power obtained with the Mahalanobis distance hardly changes ranging from $44 \%$ to $47 \%$. However, the power obtained with the other two statistics changes importantly. The power obtained with the communality statistic increases from $27 \%$ to $39 \%$ when increasing the sample size and the power obtained with the Bayes factor increases from $0 \%$ to $44 \%$.

Then we describe our comparison of software for genome scans. For the simulations obtained with the island model where there is no hierarchical population structure, the statistical power is similar for all software (Figure SI3 and SI4). Including admixed individuals hardly changes their statistical power (Figure SI3).

Then, we compare statistical power in a divergence model where adaptation took place in one of the external branches of the population divergence tree. The software pcadapt and hapflk, which account for hierarchical population structure, as well as BayeScan are the most powerful in that setting (Figure 5 and Figure SI5). The values of power in decreasing order are of $23 \%$ for BayeScan, of $20 \%$ for pcadapt, of $17 \%$ for hapflk, of $7 \%$ for $s N M F$ and of $1 \%$ for OutFLANK. When including admixed individuals, the power of hapflk and of pcadapt hardly decreases whereas the power of BayeScan decreases to $6 \%$ (Figure 5).

The last model we consider is the model of range expansion. The package pcadapt is the most powerful approach in this setting (Figure 6 and SI6). Other software also discover many true positive loci with the exception of BayeScan that provides no true discovery when the observed FDR is smaller than $50 \%$ (Figure 6 and SI6). The values of power in decreasing order are of $46 \%$ for pcadapt, of $41 \%$ for hapflk, of $37 \%$ for OutFLANK, of $30 \%$ for $s N M F$ and of $0 \%$ for BayeScan.

\section{Running time of the different software}

Last, we compare the software running times. The characteristics of the computer we used to perform comparisons are the following : OSX El Capitan 10.11.3, 2,5 GHz Intel Core i5, 8 Go $1600 \mathrm{MHz}$ DDR3. We discard BayeScan as it is too time consuming compared to other software. For instance, running BayeScan on a genotype matrix containing 150 individuals and 3,000 SNPs takes 9 hours whereas it takes less than one second with 
pcadapt. The different software were run on genotype matrices containing 300 individuals and from 500 to 50,000 SNPs. OutFLANK is the software for which the runtime increases the most rapidly with the number of markers. OutFLANK takes around 25 minutes to analyse 50,000 SNPs (Figure SI7). For the other 3 software (hapflk, pcadapt, sNMF), analyzing 50,000 SNPs takes less than 3 minutes.

\section{Discussion}

The R package pcadapt implements a fast method to perform genome scans with next generation sequencing data. It can handle datasets where population structure is continuous or datasets containing admixed individuals. It can handle missing data as well as pooled sequencing data. The 2.0 and later versions of the $\mathrm{R}$ package implements a robust Mahalanobis distance as a test statistic. When hierarchical population structure occurs, Mahalanobis distance provides more powerful genome scans compared to the communality statistic that was implemented in the first version of the package (Duforet-Frebourg et al., 2016). In the divergence model, adaptation occurs along an external branch of the divergence tree that corresponds to the second principal component. When outlier SNPs are not related to the first principal component, the Mahalanobis distance provides a better ranking of the SNPs compared to the communality statistic.

Simulations show that the $\mathrm{R}$ package pcadapt compares favorably to other software of genome scans. When data were simulated under an island model, population structure is not hierarchical because genetic differentiation is the same for all pairs of populations. Statistical power and control of the FDR were similar for all software. In presence of hierarchical population structure (divergence model) where genetic differentiation varies between pairs of populations, the ranking of the SNPs is software dependent. The software pcadapt and hapflk provide the most powerful scans whether or not simulations include admixed individuals. OutFLANK implements a $F_{S T}$ statistic and because adaptation does not correspond to the most differentiated populations, it fails to capture adaptive SNPs (Figure 5) (Bonhomme et al., 2010; Duforet-Frebourg et al., 2016). BayeScan does not assume equal differentiation between all pairs of populations, which may explain why it has a good statistical power for the divergence model. However its statistical power is severely impacted by the presence of admixed individuals because its power decreases from $24 \%$ to $6 \%$ (Figure 5). Understanding why BayeScan is severely impacted by admixture is 
out of the scope of this paper. In the range expansion model, BayeScan returns many null $q$-values (between 376 and 809 SNPs out of 9,899 neutral and 100 adaptive SNPs) such that the observed FDR is always larger than $50 \%$. Overall, we find that pcadapt and hapflk provides comparable statistical power. Compared to other software, they provide optimal or near optimal ranking of the SNPs in different scenarios including hierarchical population structure and admixed individuals. The main difference between the two software concerns the control of the FDR because hapflk is found to be more conservative.

Because NGS data become more and more massive, careful numerical implementation is crucial. There are different options to implement PCA and pcadapt uses a numerical routine based on the computation of the covariance matrix $\Omega$. The algorithmic complexity to compute the covariance matrix is proportional to $p n^{2}$ where $p$ is the number of markers and $n$ is the number of individuals. The computation of the first $K$ eigenvectors of the covariance matrix $\Omega$ has a complexity proportional to $n^{3}$. This second step is usually more rapid than the computation of the covariance because the number of markers is usually large compared to the number of individuals. In brief, computing the covariance matrix $\Omega$ is by far the most costly operation when computing principal components. Although we have implemented PCA in $C$ to obtain fast computations, an improvement in speed could be envisioned for future versions. When the number of individuals becomes large (e.g. $n \geq 10,000$ ), there are faster algorithms to compute principal components (Halko et al., 2011; Abraham and Inouye, 2014). In addition to running time, numerical implementations also impact the effect of missing data on principal components (Dray and Josse, 2015). Achieving a good tradeoff between fast computations and accurate evaluation of population structure in the face of large amount of missing data is a challenge for modern numerical methods in molecular ecology. 


\begin{tabular}{|c|c|c|c|c|c|}
\hline Test statistic & Pop. structure & Language & Command line & Versions of the R package & Ref. \\
\hline Bayes factor & Factor model & C & PCAdapt & NA & Duforet-Frebourg et al. (2014) \\
Communality & PCA & C and R & PCAdapt fast & Duforet-Frebourg et al. (2016) \\
Mahalanobis dist. & PCA & R & NA & $2 . x$ and 3.x & This paper \\
\hline
\end{tabular}

TABLE 1 - Summary of the different statistical methods and implementations of pcadapt. Pop. structure stands for population structure and dist. stands for distance.

\begin{tabular}{|l|l|l|l|l|}
\hline & Individuals & SNPs & Adaptive SNPs & Simulations \\
\hline Island model & 150 & 472 & 27 & 35 \\
\hline Divergence model & 150 & 3000 & 100 & 6 \\
\hline Island model (hybrids) & 150 & 472 & 30 & 27 \\
\hline Divergence model (hybrids) & 150 & 3000 & 100 & 9 \\
\hline Range expansion & 1200 & 9999 & 99 & 6 \\
\hline
\end{tabular}

TABLE 2 - Summary of the simulations. The table above shows the average number of individuals, of SNPs, of adaptive markers and the total number of simulations per scenario. 

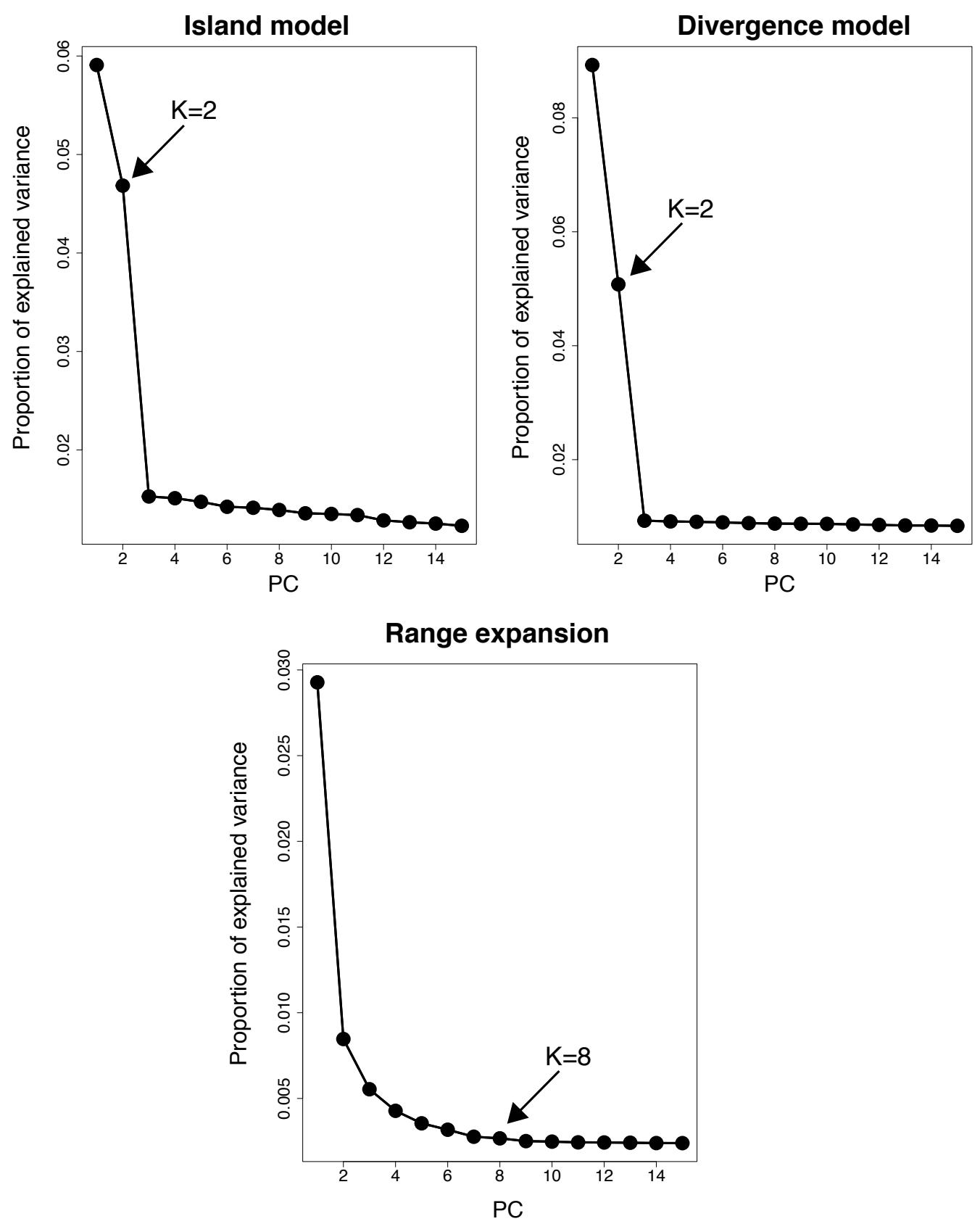

Figure 1 - Determining $K$ with the screeplot. To choose $K$, we recommend to use Cattell's rule that states that components corresponding to eigenvalues to the left of the straight line should be kept. According to Cattell's rule, the eigenvalues that correspond to random variation lie on the straight line whereas the ones corresponding to population structure depart from the line. For the island and divergence model, the choice of $\mathrm{K}$ is evident. For the model or range expansion, a value of $K$ between 5 and 8 is compatible with Cattell's rule. 


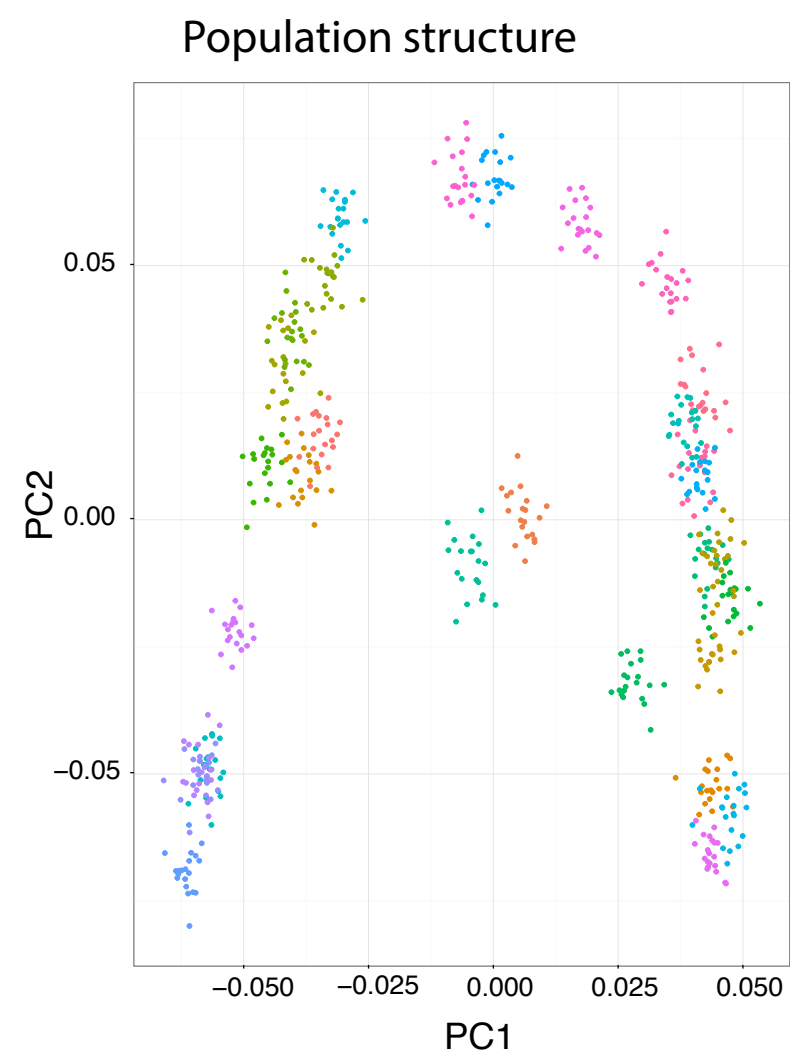

Distribution of $p$-values

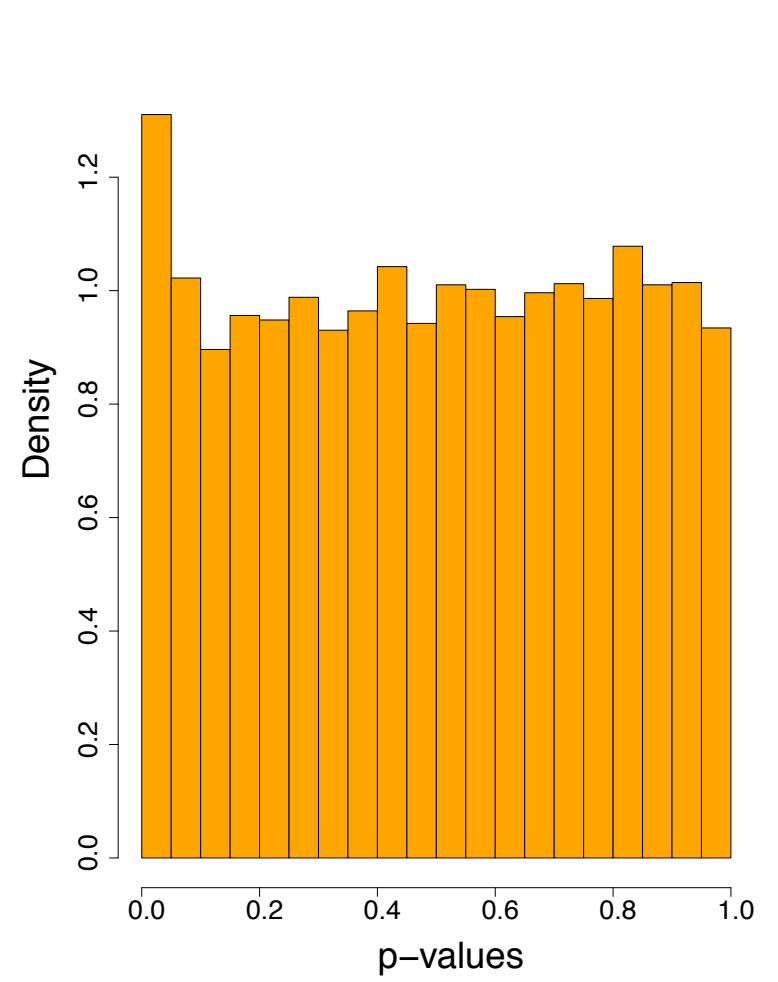

FIGURE 2 - Population structure (first 2 principal components) and distribution of $p$-value obtained with pcadapt for a simulation of range expansion. $P$-values are well calibrated because they are distributed as a mixture of a uniform distribution and of a peaky distribution around 0 , which corresponds to outlier loci. In the left panel, each color corresponds to individuals sampled from the same population. 


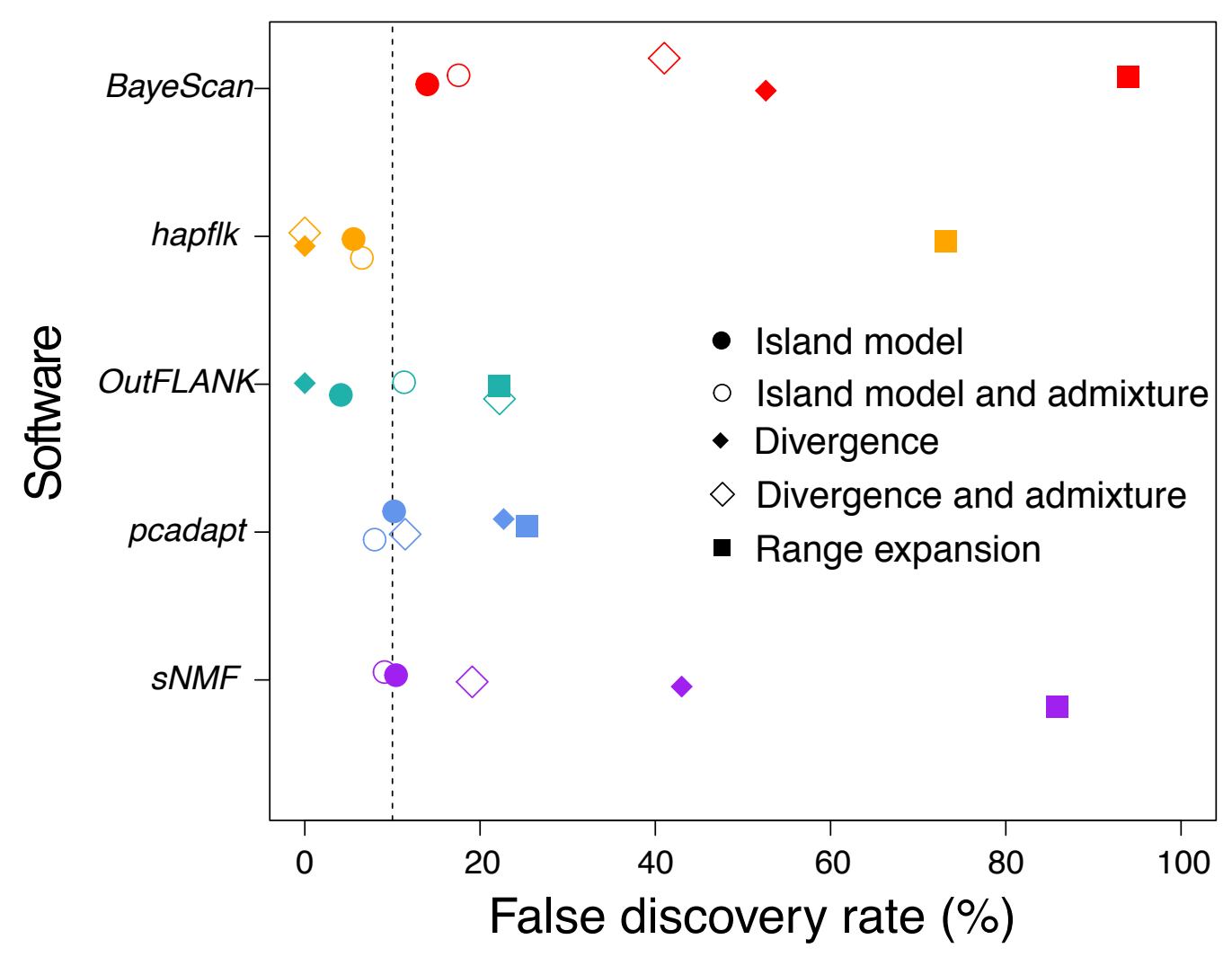

FiguRE 3 - Control of the FDR for different software of genome scans. We find that the median proportion of false discoveries is around the nominal FDR set at $10 \%(6 \%$ for hapflk, $11 \%$ for both OutFLANK and pcadapt, and $19 \%$ for $s N M F$ ) with the exception of BayeScan that generates $41 \%$ of false discoveries. 


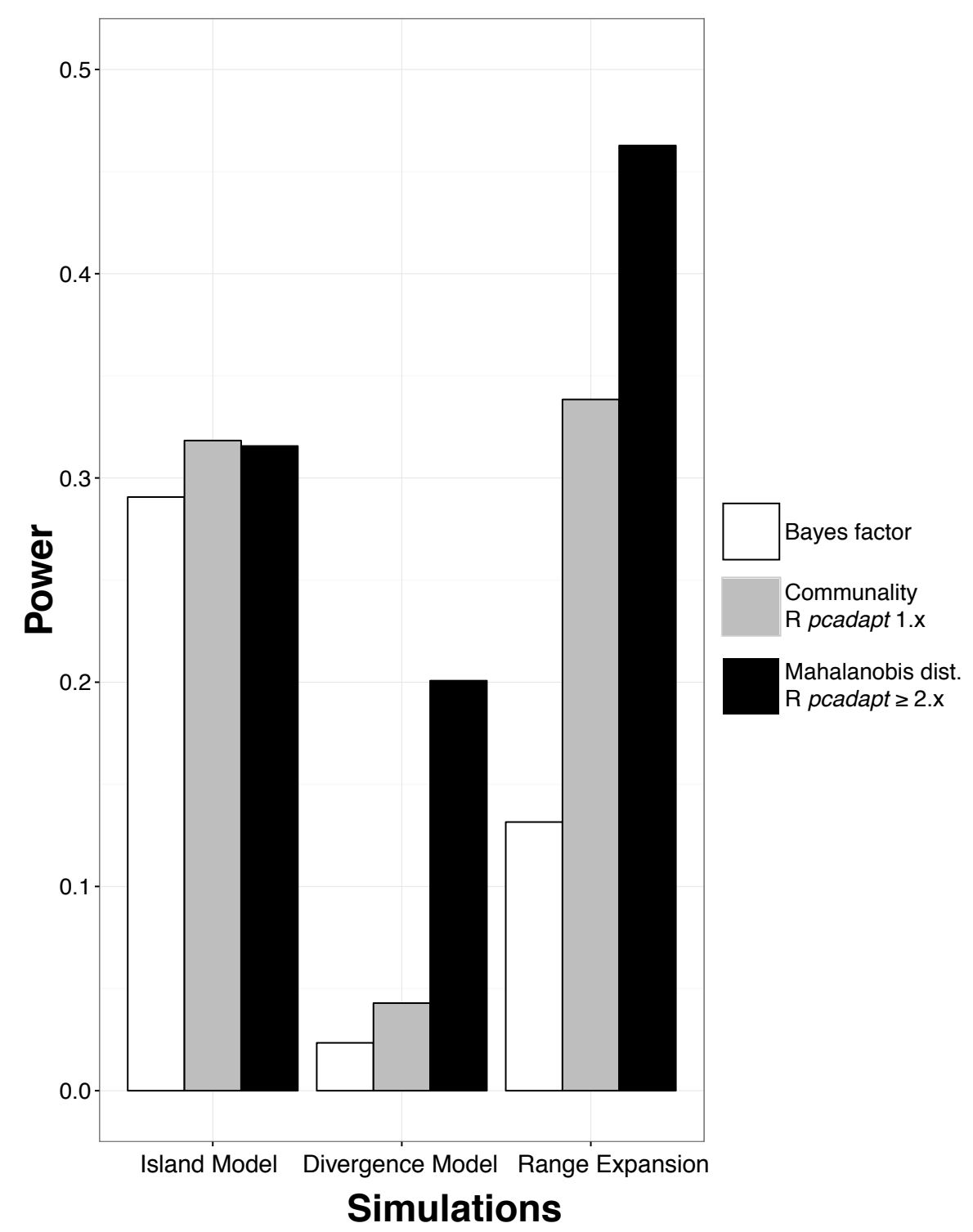

FIgURE 4 - Comparison of statistical power for the different test statistics that have been implemented in pcadapt (Table 1). Bayes factors corresponds to the test statistics implemented in the Bayesian version of pcadapt (Duforet-Frebourg et al., 2014); the communality statistic was the default statistic in version 1.x of the $\mathrm{R}$ package pcadapt (Duforet-Frebourg et al., 2016), and Mahalanobis distances are available since the release of the 2.0 version of the package. When there is hierarchical population structure (divergence model and range expansion), the Mahalanobis distance provides more powerful genome scans compared to the test statistic previously implemented in pcadapt. The abbreviation dist. stands for distance. Statistical power is averaged over the observed proportion of false discoveries (ranging between $0 \%$ and 50\%). 


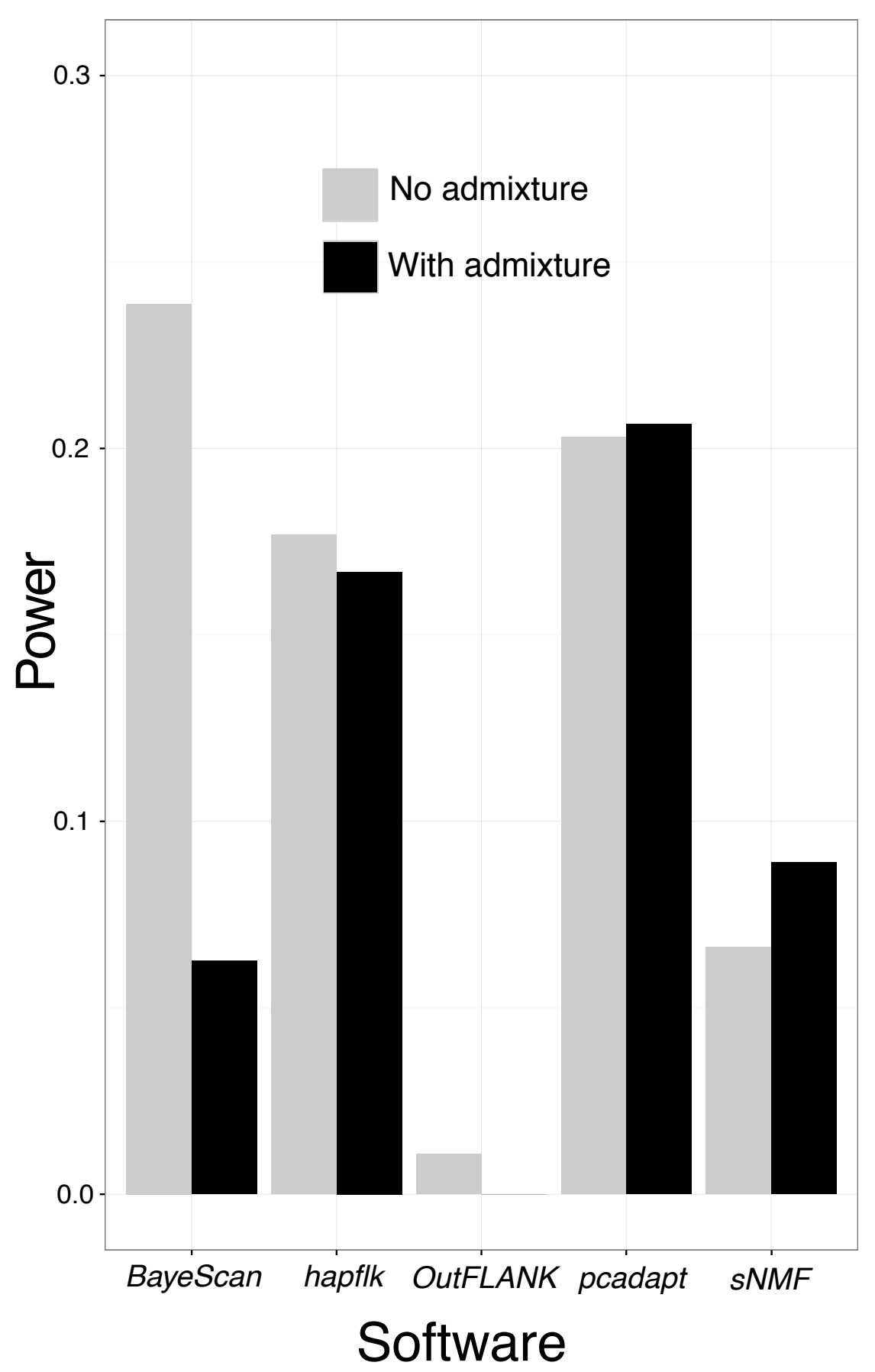

FigURE 5 - Statistical power averaged over the expected proportion of false discoveries (ranging between $0 \%$ and $50 \%$ ) for the divergence model with 3 populations. We assume that adaptation took place in an external branch that follows the most recent population divergence event. 


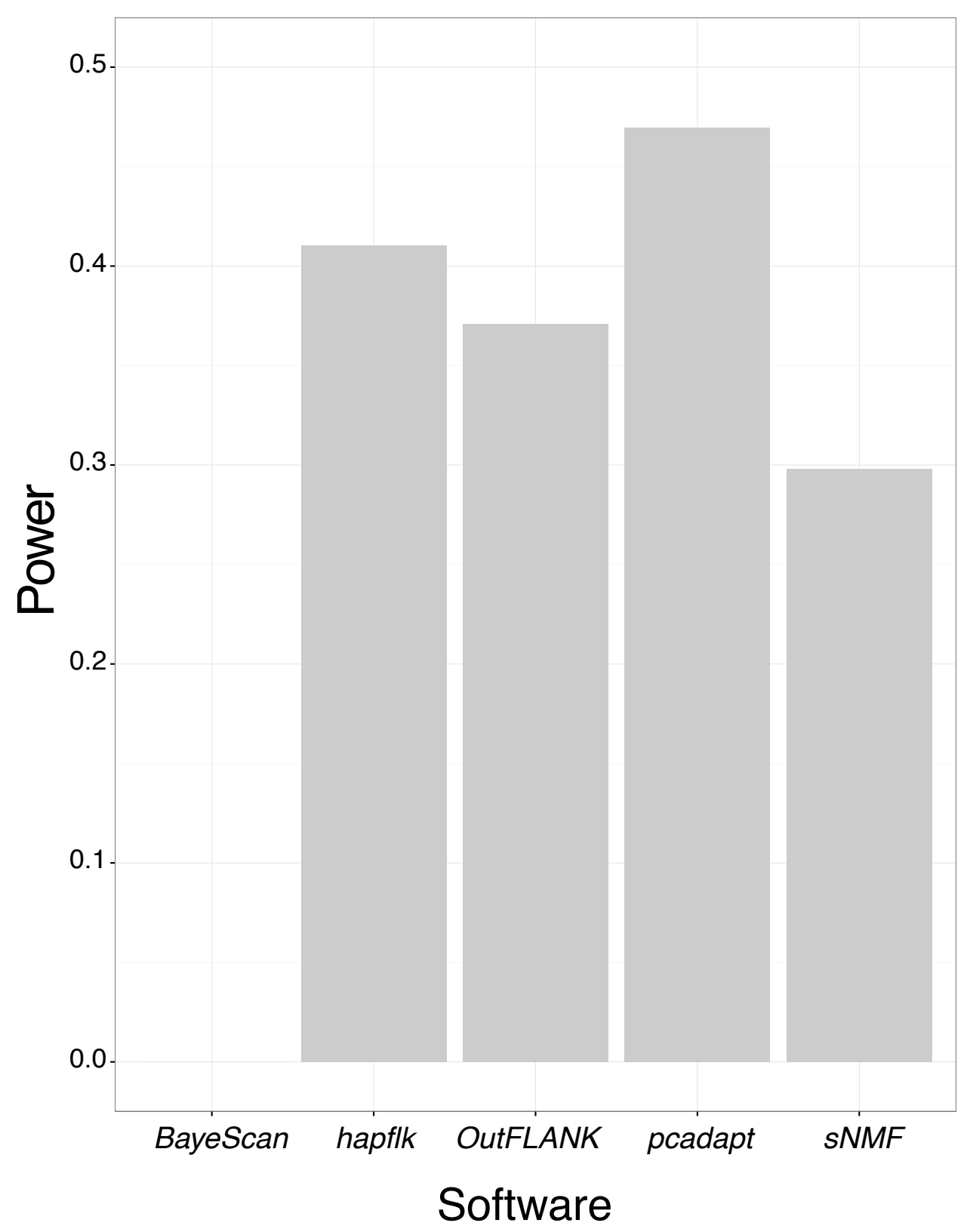

FIGURE 6 - Statistical power averaged over the expected proportion of false discoveries (ranging between $0 \%$ and 50\%) for a range expansion model with two refugia. Adaptation took place during the recolonization event. 


\section{${ }_{371}$ Acknowledgements}

372 This work has been supported by the LabEx PERSYVAL-Lab (ANR-11-LABX-0025373 01) and the ANR AGRHUM project (ANR-14-CE02-0003-01). 
374

\section{References}

Abraham, G. and M. Inouye, 2014. Fast principal component analysis of large-scale genome-wide data. PloS one 9 :e93766.

Arnold, B., R. Corbett-Detig, D. Hartl, and K. Bomblies, 2013. RADseq underestimates diversity and introduces genealogical biases due to nonrandom haplotype sampling. Molecular ecology $22: 3179-3190$.

Bazin, E., K. J. Dawson, and M. A. Beaumont, 2010. Likelihood-free inference of population structure and local adaptation in a bayesian hierarchical model. Genetics $185: 587-602$.

Bierne, N., D. Roze, and J. J. Welch, 2013. Pervasive selection or is it...? why are fst outliers sometimes so frequent? Molecular ecology 22 :2061-2064.

Bonhomme, M., C. Chevalet, B. Servin, S. Boitard, J. Abdallah, S. Blott, and M. SanCristobal, 2010. Detecting selection in population trees : the Lewontin and Krakauer test extended. Genetics $186: 241-262$.

Cattell, R. B., 1966. The scree test for the number of factors. Multivariate behavioral research $1: 245-276$.

Chen, G.-B., S. H. Lee, Z.-X. Zhu, B. Benyamin, and M. R. Robinson, 2016. Eigengwas : finding loci under selection through genome-wide association studies of eigenvectors in structured populations. Heredity $117: 51-61$.

Devlin, B. and K. Roeder, 1999. Genomic control for association studies. Biometrics $55: 997-1004$.

Dray, S. and J. Josse, 2015. Principal component analysis with missing values : a comparative survey of methods. Plant Ecology 216 :657-667.

Duforet-Frebourg, N., E. Bazin, and M. G. B. Blum, 2014. Genome scans for detecting footprints of local adaptation using a bayesian factor model. Molecular biology and evolution 31 :2483-2495. 
Duforet-Frebourg, N., K. Luu, G. Laval, E. Bazin, and M. G. B. Blum, 2016. Detecting genomic signatures of natural selection with principal component analysis : application to the 1000 genomes data. Molecular biology and evolution 33 :1082-1093.

Excoffier, L., T. Hofer, and M. Foll, 2009. Detecting loci under selection in a hierarchically structured population. Heredity $103: 285-298$.

Foll, M. and O. Gaggiotti, 2008. A genome-scan method to identify selected loci appropriate for both dominant and codominant markers : a Bayesian perspective. Genetics 180 :977-993.

François, O., H. Martins, K. Caye, and S. D. Schoville, 2016. Controlling false discoveries in genome scans for selection. Molecular ecology 25 :454-469.

Frichot, E., F. Mathieu, T. Trouillon, G. Bouchard, and O. François, 2014. Fast and efficient estimation of individual ancestry coefficients. Genetics 196 :973-983.

Galinsky, K. J., G. Bhatia, P.-R. Loh, S. Georgiev, S. Mukherjee, N. J. Patterson, and A. L. Price, 2016. Fast principal components analysis reveals independent evolution of adh1b gene in Europe and East Asia. American Journal of Human Genetics 98 :456472 :018143.

Gautier, M., K. Gharbi, T. Cezard, J. Foucaud, C. Kerdelhué, P. Pudlo, J.-M. Cornuet, and A. Estoup, 2013. The effect of RAD allele dropout on the estimation of genetic variation within and between populations. Molecular Ecology 22 :3165-3178.

Halko, N., P.-G. Martinsson, and J. A. Tropp, 2011. Finding structure with randomness : Probabilistic algorithms for constructing approximate matrix decompositions. SIAM review $53: 217-288$.

Hao, W., M. Song, and J. D. Storey, 2016. Probabilistic models of genetic variation in structured populations applied to global human studies. Bioinformatics 32 :713-721.

Jackson, D. A., 1993. Stopping rules in principal components analysis : a comparison of heuristical and statistical approaches. Ecology Pp. 2204-2214.

Lange, K., J. C. Papp, J. S. Sinsheimer, and E. M. Sobel, 2014. Next generation statistical genetics : Modeling, penalization, and optimization in high-dimensional data. Annual review of statistics and its application $1: 279$. 
Lewontin, R. and J. Krakauer, 1973. Distribution of gene frequency as a test of the theory of the selective neutrality of polymorphisms. Genetics 74 :175-195.

Lotterhos, K. E. and M. C. Whitlock, 2015. The relative power of genome scans to detect local adaptation depends on sampling design and statistical method. Molecular ecology $24: 1031-1046$.

Maronna, R. A. and R. H. Zamar, 2012. Robust estimates of location and dispersion for high-dimensional datasets. Technometrics 44 :307-317.

Martins, H., K. Caye, K. Luu, M. G. Blum, and O. Francois, 2016. Identifying outlier loci in admixed and in continuous populations using ancestral population differentiation statistics. bioRxiv P. 054585.

Patterson, N., A. L. Price, and D. Reich, 2006. Population structure and eigenanalysis. PLoS genet 2 :e190.

Peng, B. and M. Kimmel, 2005. simuPOP : a forward-time population genetics simulation environment. Bioinformatics $21: 3686-3687$.

Pritchard, J. K., M. Stephens, and P. Donnelly, 2000. Inference of population structure using multilocus genotype data. Genetics 155 :945-959.

R Core Team, 2015. R : A Language and Environment for Statistical Computing. R Foundation for Statistical Computing, Vienna, Austria. URL https : //www.R-project.org.

Storey, J. D. and R. Tibshirani, 2003. Statistical significance for genomewide studies. Proceedings of the National Academy of Sciences $100: 9440-9445$.

Waples, R. S. and O. Gaggiotti, 2006. Invited review : What is a population? an empirical evaluation of some genetic methods for identifying the number of gene pools and their degree of connectivity. Molecular ecology 15 :1419-1439.

Whitlock, M. C. and K. E. Lotterhos, 2015. Reliable detection of loci responsible for local adaptation : Inference of a null model through trimming the distribution of fst. The American naturalist 186 :S24-36. 


\section{${ }_{455}$ Data Accessibility}

456 Island and divergence model data : doi :10.5061/dryad.8290n

${ }_{457}$ Range expansion simulated data : doi :10.5061/dryad.mh67v. Files :

2R_R30_1351142954_453_2_NumPops $=30 \_N u m I n d=20$

2R_R30_1351142954_453_2_NumPops $=30 \_N u m I n d=60$

2R_R30_1351142970_988_6_NumPops $=30 \_N u m I n d=20$

2R_R30_1351142970_988_6_NumPops $=30 \_N u m I n d=60$

2R_R30_1351142986_950_10_NumPops $=30 \_N u m I n d=20$

2R_R30_1351142986_950_10_NumPops=30_NumInd $=60$ 


\section{Island model}

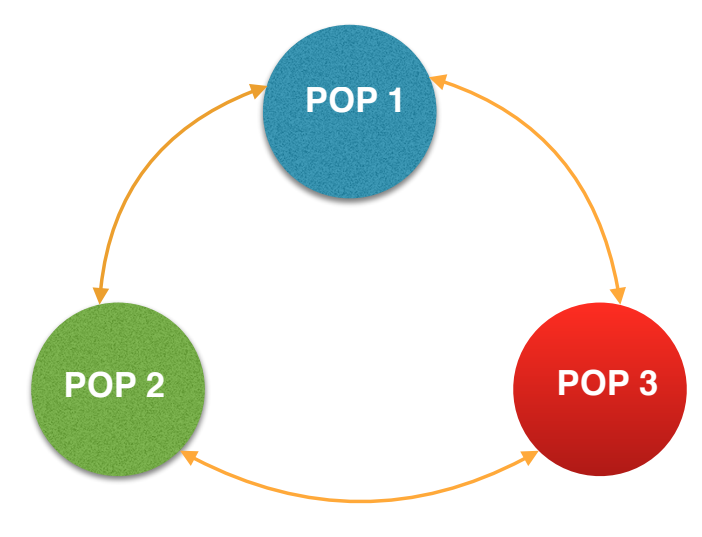

\section{Divergence model}

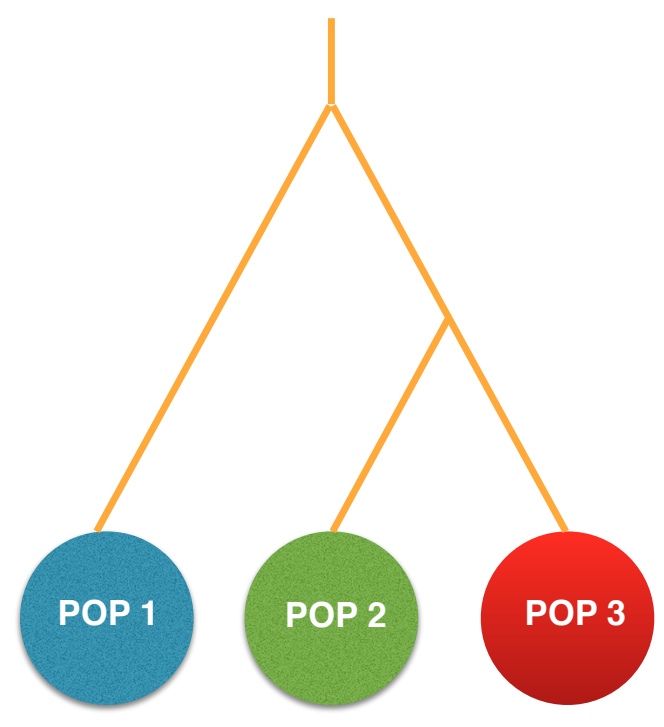

FIGURE SI1 - Schematic description of the island and divergence model. For the island model, adaptation occurs simultaneously in each population. For the island model, adaptation takes place in the branch leading to the second population. 

aCC-BY-NC 4.0 International license.

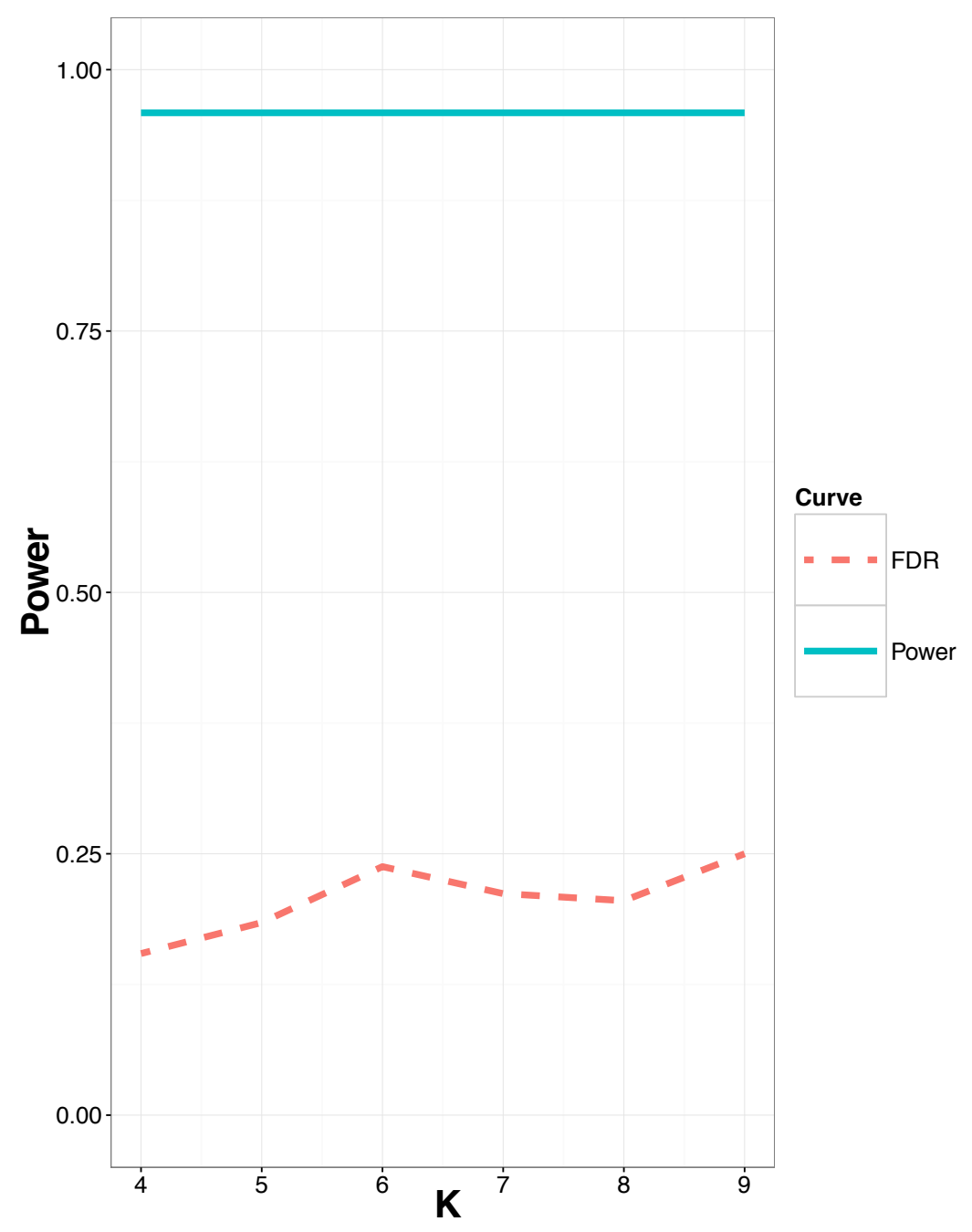

FiguRE SI2 - Proportion of false discoveries and statistical power as a function of the number of principal components in a model of range expansion. 

aCC-BY-NC 4.0 International license.

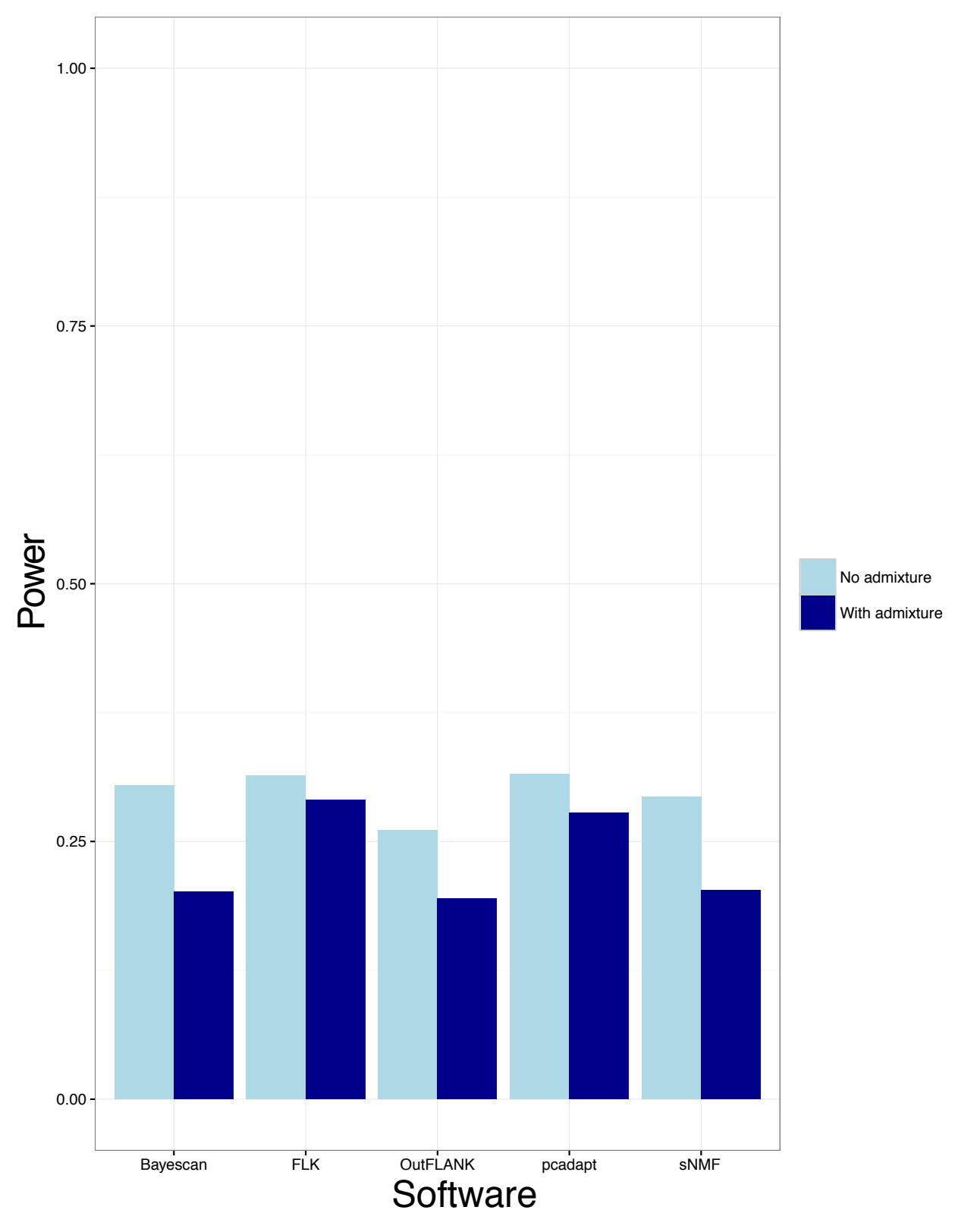

FIGURE SI3 - Statistical power averaged over the expected proportion of false discoveries (ranging between 0\% and 50\%) for the island model. 

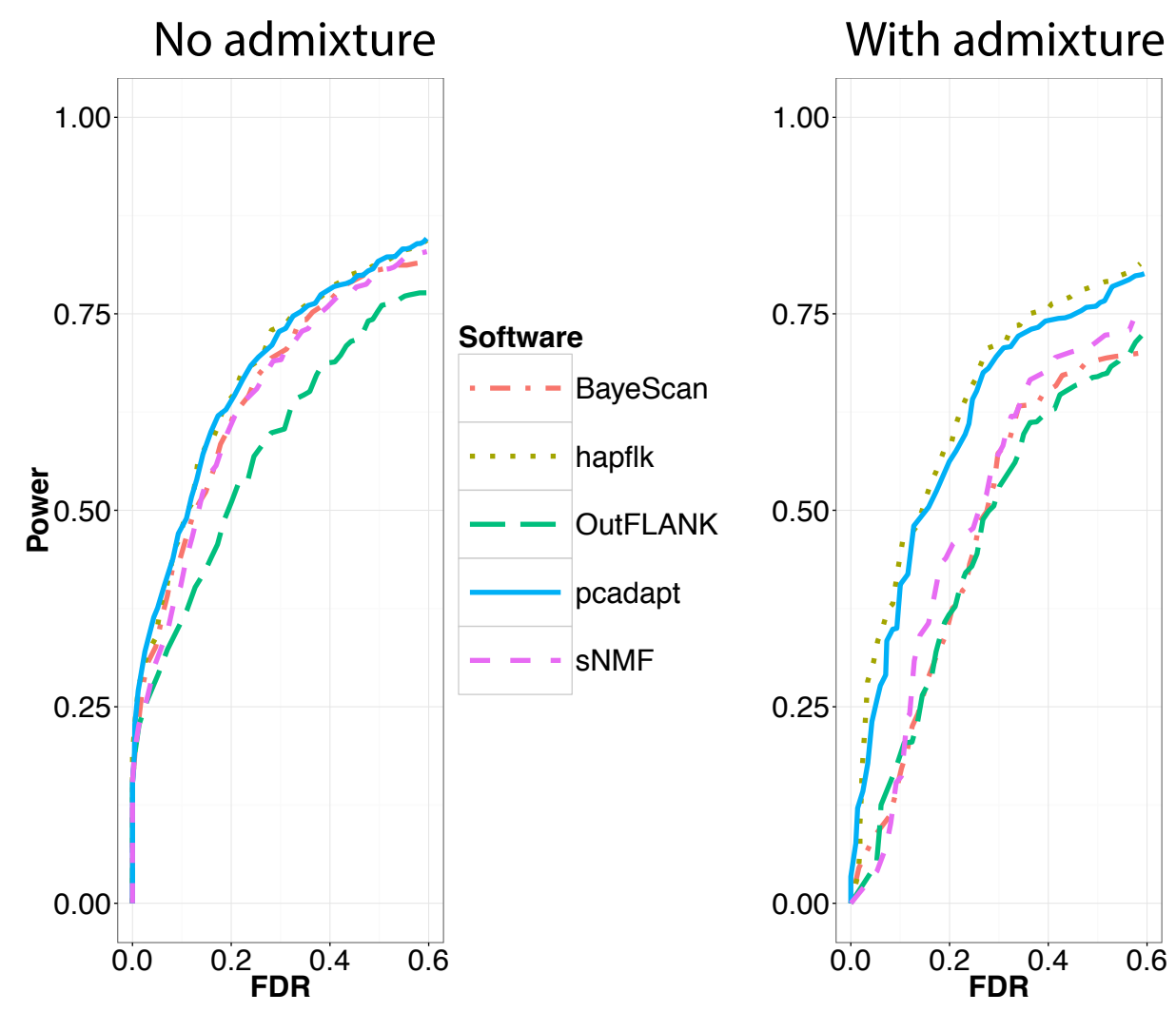

FigURE SI4 - Statistical power as a function of the proportion of false discoveries for the island model. 

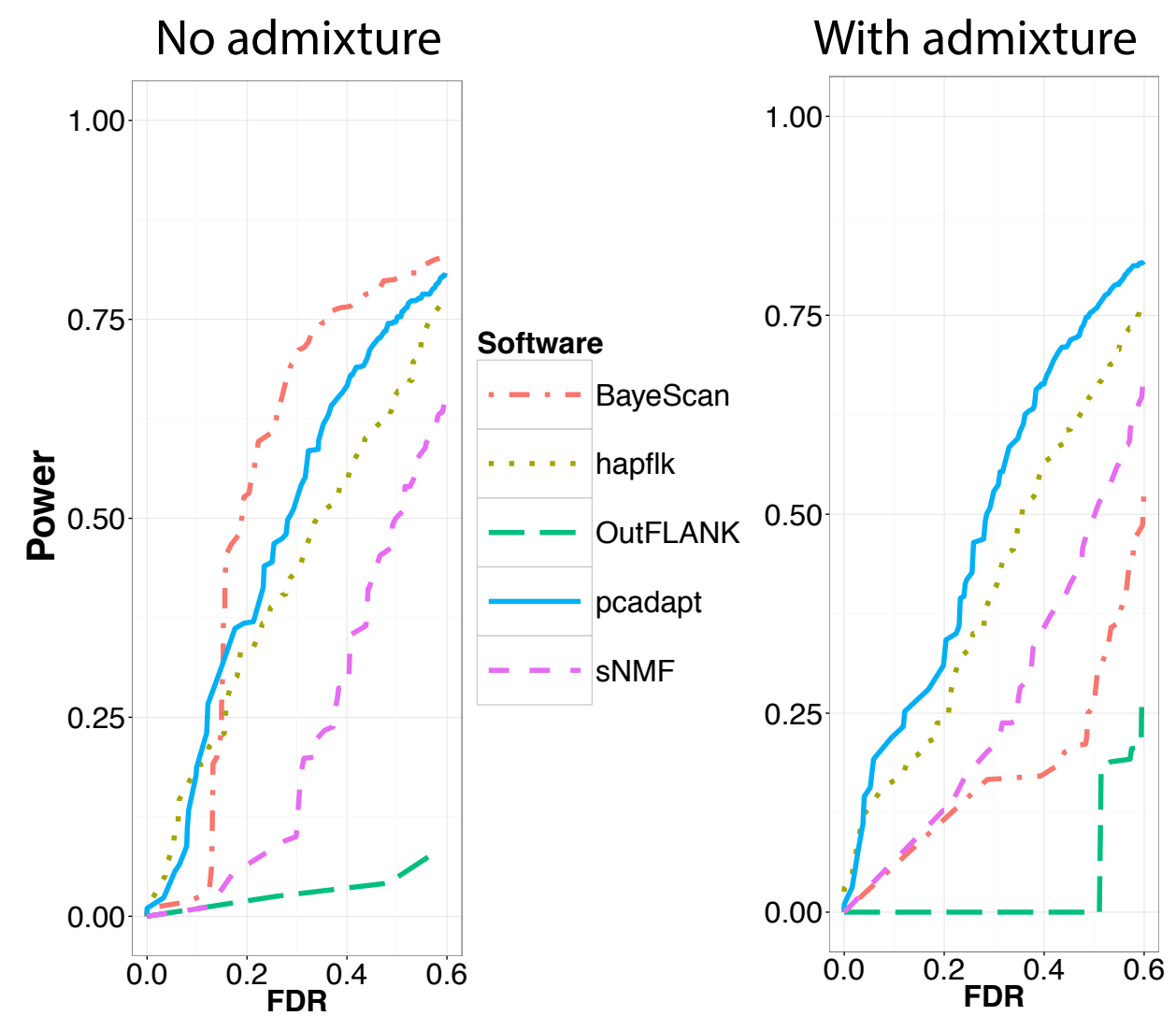

FIGURE SI5 - Statistical power as a function of the proportion of false discoveries for the divergence model. 


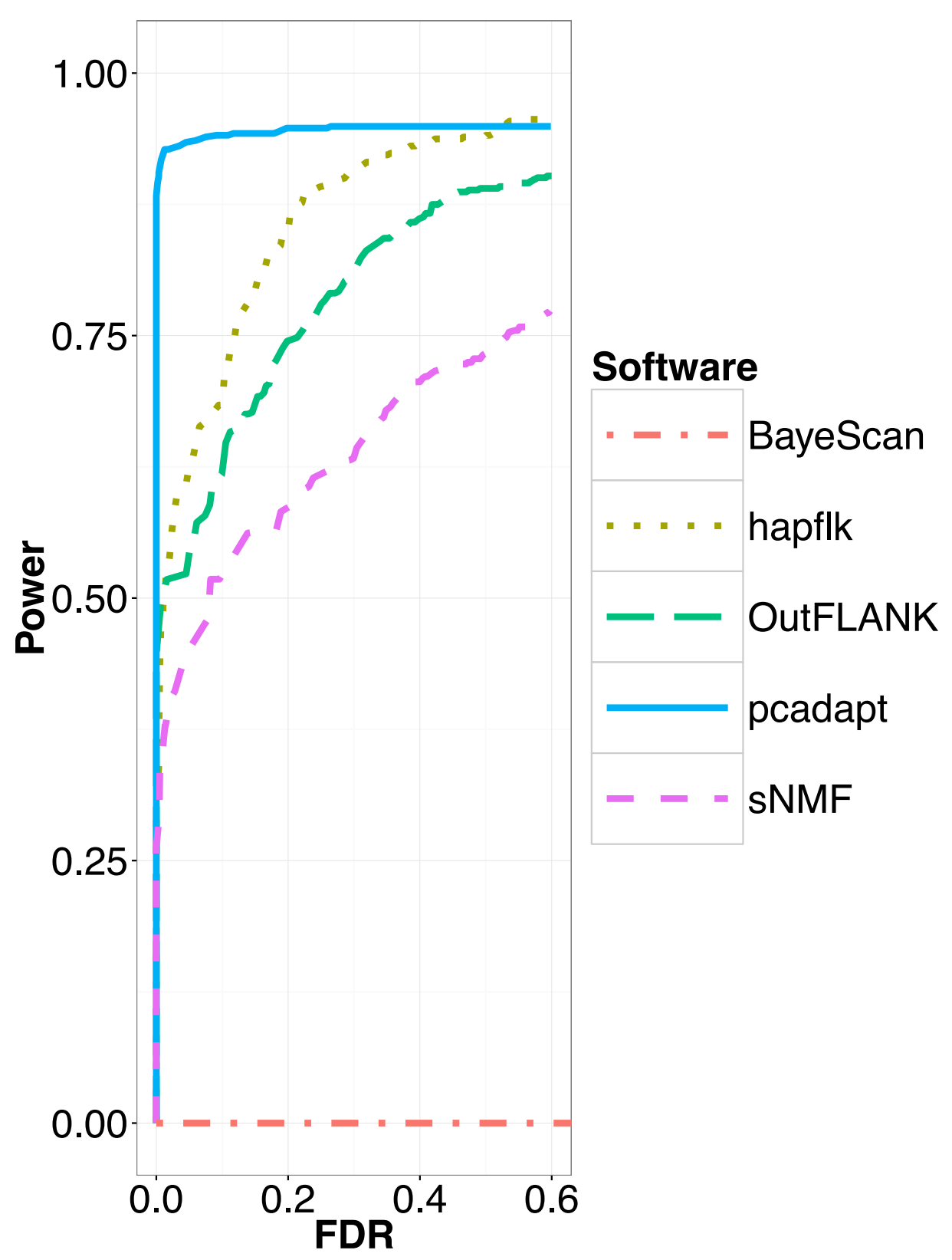

FIGURE SI6 - Statistical power as a function of the proportion of false discoveries for the model of range expansion. 


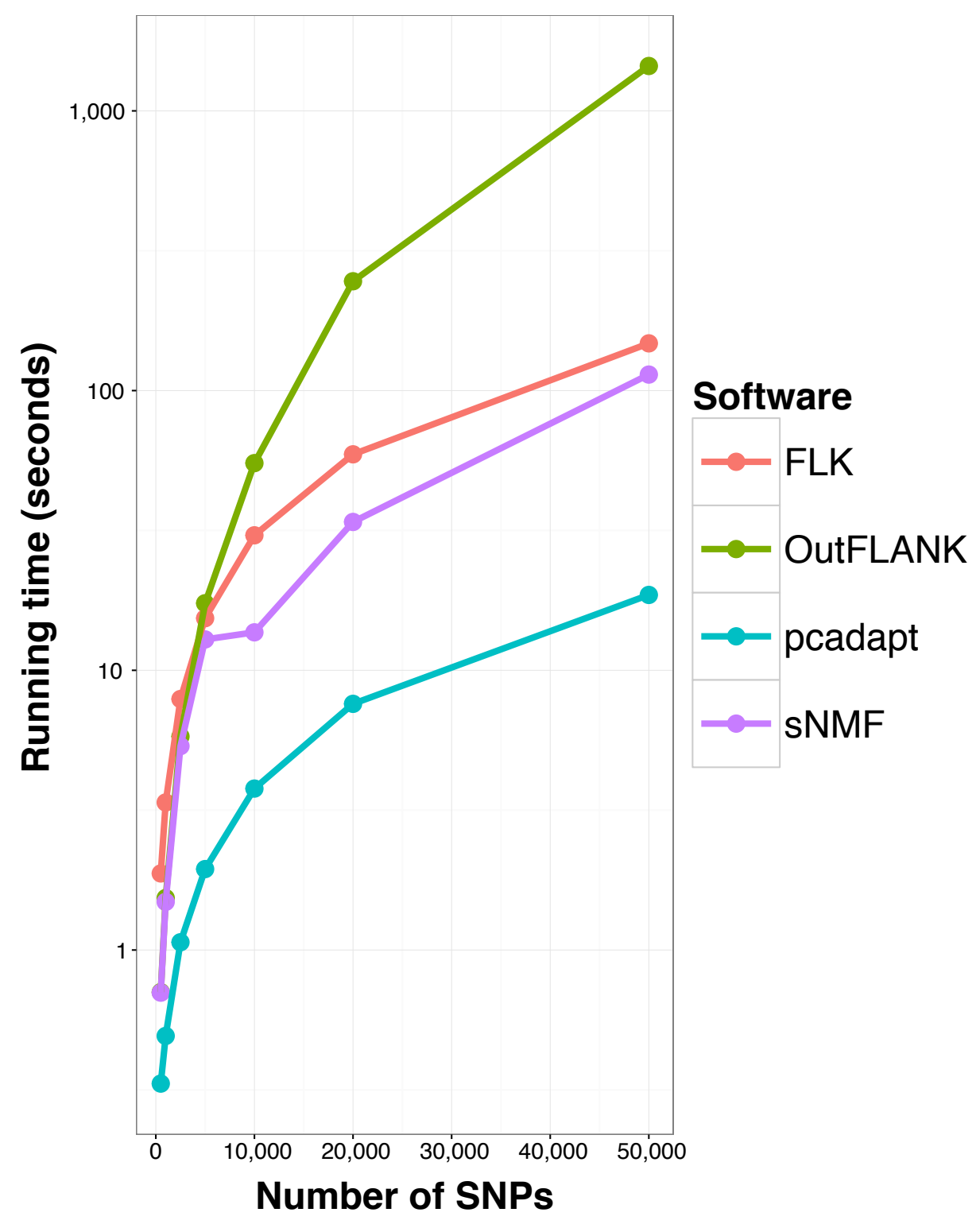

FiguRE SI7 - Running times of the different software. The different software were run on genotype matrices containing 300 individuals and from 500 to 50,000 SNPs. The characteristics of the computer we used to perform comparisons is the following : OSX El Capitan 10.11.3, 2,5 GHz Intel Core i5, 8 Go 1600 MHz DDR3. 